\title{
Local load sharing fiber bundles with a lower cutoff of strength disorder
}

\author{
Frank Raischel, ${ }^{1, *}$ Ferenc Kun, ${ }^{2}$ and Hans J. Herrmann ${ }^{1,3}$ \\ ${ }^{1}$ ICP, University of Stuttgart, Pfaffenwaldring 27, D-70569 Stuttgart, Germany \\ ${ }^{2}$ Department of Theoretical Physics, University of Debrecen, P.O. Box:5, H-4010 Debrecen, Hungary \\ ${ }^{3}$ IfB, ETH, Schafmattstr. 6, CH-8093 Zürich, Switzerland
}

(Received 12 July 2006; published 27 September 2006)

\begin{abstract}
We study the failure properties of fiber bundles with a finite lower cutoff of the strength disorder varying the range of interaction between the limiting cases of completely global and completely local load sharing. Computer simulations revealed that at any range of load redistribution there exists a critical cutoff strength where the macroscopic response of the bundle becomes perfectly brittle, i.e., linearly elastic behavior is obtained up to global failure, which occurs catastrophically after the breaking of a small number of fibers. As an extension of recent mean field studies [Phys. Rev. Lett. 95, 125501 (2005)], we demonstrate that approaching the critical cutoff, the size distribution of bursts of breaking fibers shows a crossover to a universal power law form with an exponent $3 / 2$ independent of the range of interaction.
\end{abstract}

DOI: 10.1103/PhysRevE.74.035104

PACS number(s): 46.50.+a, 62.20.Mk, 81.40.Np

In engineering constructions solids are subjected to various types of external loads and typically fail when the load exceeds a critical value. Monitoring stressed systems and forecasting an imminent failure event is of enormous importance due to the possible material and human costs. In spite of the large amount of experimental and theoretical effort that has been undertaken over the past decades, there is no comprehensive understanding of failure phenomena, which is also reflected by the absence of reliable prediction methods. Materials of low disorder typically fail in a "one-crack" way, where the main problem is to prevent crack initiation and propagation. However, the failure of highly disordered materials proceeds in bursts of local breaking events that can be recorded as acoustic signals. Experiments on a large variety of materials have revealed that in crackle noise spectra accompanying quasistatic fracture of disordered materials, the amplitude and duration of signals and the waiting time between them are characterized by power law distributions over a broad range [1-3]. Quantitative changes of the burst activity when approaching the critical load could be precursors of catastrophic failure and may serve as the basis for forecasting techniques.

In the framework of discrete models of the fracture of disordered materials, bursts can be identified as trails of correlated breakings of the microscopic constituents of the model. Fiber bundle models consist of a parallel bundle of fibers with identical linearly elastic behavior and randomly distributed breaking thresholds [4-6]. Under an increasing external load, each fiber breaking is followed by a load redistribution over the remaining intact fibers, which may trigger avalanches of correlated breaking events analogous to crackling noise in experiments. Assuming global load sharing (GLS), it has been shown that the distribution of avalanche sizes has a universal power law behavior with an exponent $5 / 2$ [7]. In the other extreme of local load sharing (LLS), redistributing the load solely over the closest neighborhood of fibers, the avalanche distribution appears also to be a power law but with a higher exponent $\approx 9 / 2[7,8]$. For

\footnotetext{
*Electronic address: raischel@ica1.uni-stuttgart.de
}

global load sharing it has recently been pointed out that the distribution of burst sizes significantly changes if the weak fibers are removed from the bundle: if the strength distribution of fibers has a finite lower cutoff, or analogously, if the recording of avalanches starts after the breaking of the weak elements, the burst size distribution is found to show a crossover to another power law with a significantly lower exponent $3 / 2[9,10]$. The effective range of interaction in real materials may have large variations [11], therefore, in order to use the crossover effect of burst sizes in forecasting of imminent failure, its robustness with respect to the range of interaction must be explored.

In the present paper we extend recent mean field studies of the effect of the lower cutoff of fiber strength on the failure process of fiber bundle models $[9,10]$ by continuously varying the range of load sharing between the limiting cases of completely global load sharing and the very localized one [11]. We show that at any range of interaction there exists a critical value of the cutoff strength above which the global response of the bundle becomes perfectly brittle, as in the GLS case [6]. We demonstrate that the crossover of the avalanche size distribution to a power law of an exponent $3 / 2$, when approaching the critical cutoff strength, is independent of the range of interaction. Our results support the usage of the crossover phenomenon of burst sizes in forecasting techniques of imminent failure.

We consider a parallel bundle of fibers organized on a square lattice of size $L \times L$. The fibers are assumed to have linearly elastic behavior with identical Young modulus $E$ up to a randomly distributed breaking threshold. For simplicity, the failure thresholds $\sigma_{\text {th }}$ are assumed to have a uniform distribution between a lower cutoff strength $\sigma_{L}$ and one with the probability density function $p\left(\sigma_{\mathrm{th}}\right)[12]$,

$$
p\left(\sigma_{\mathrm{th}}\right)= \begin{cases}\frac{1}{1-\sigma_{L}} & \text { for } \sigma_{L} \leqslant \sigma_{t h} \leqslant 1, \\ 0 & \text { otherwise. }\end{cases}
$$

Under an increasing external load the fibers break when the load on them exceeds the local threshold value $\sigma_{\text {th }}^{i}$, where $i=1, \ldots, N$ and $N=L^{2}$ denotes the number of fibers. Due to 

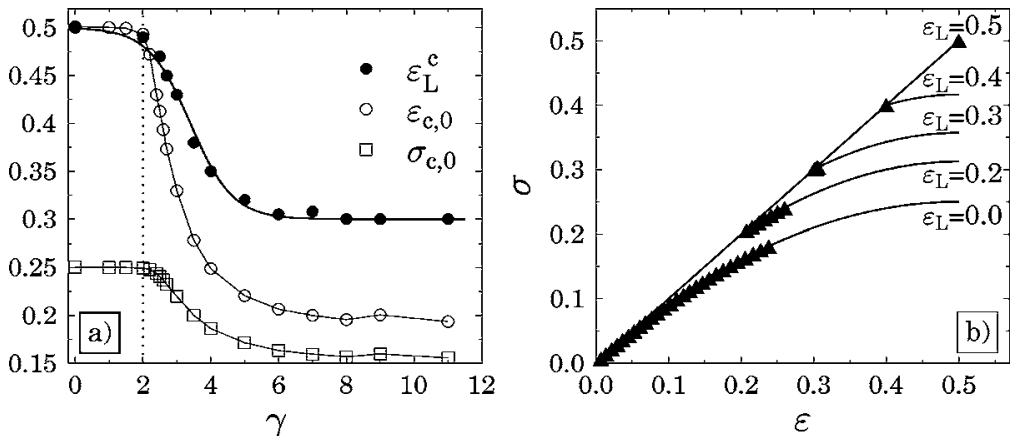

FIG. 1. (a) Failure stress $\sigma_{c}$ and strain $\varepsilon_{c}$ of the fiber bundle model at zero cutoff $\varepsilon_{L}=0$ compared to the critical cutoff $\varepsilon_{L}^{c}$ as a function of $\gamma$. (b) Constitutive curves for GLS $\gamma=0$ (lines) compared to the case of $\gamma=5$ (triangles) at different values of $\varepsilon_{L}$. the linearly elastic behavior, the failure thresholds $\sigma_{\text {th }}^{i}$ can also be expressed in terms of deformation $\varepsilon_{\mathrm{th}}^{i}=\sigma_{\mathrm{th}}^{i} / E$ with the cutoff strength $\varepsilon_{L}=\sigma_{L} / E$. After a failure event, the remaining intact fibers must take over the load of the failed one. In order to give a realistic description of the load redistribution in FBMs, we recently introduced a load transfer function of the form

$$
\sigma_{\mathrm{add}}=\frac{1}{Z} r_{i j}^{-\gamma}
$$

where $\sigma_{\text {add }}$ denotes the additional load fiber $i$ received after the breaking of fiber $j[11]$. The load increment $\sigma_{\text {add }}$ decreases as a power law of the distance $r_{i j}$ from the failed fiber, where the exponent $\gamma$ is considered to be a free parameter of the model. The exponent $\gamma$ can take any values between 0 and $\infty$ controlling the effective range of load redistribution between the limiting cases of completely global $\gamma=0$ and completely localized load redistribution $\gamma \rightarrow \infty$ [11].

Under perfectly global load sharing $\gamma=0$ the macroscopic constitutive equation of the system can be cast in a simple analytic form

$$
\sigma(\varepsilon)= \begin{cases}E \varepsilon & \text { for } E \varepsilon \leqslant \sigma_{L}, \\ E \varepsilon \frac{1-E \varepsilon}{1-E \varepsilon_{L}} & \text { for } \sigma_{L} \leqslant E \varepsilon \leqslant 1,\end{cases}
$$

where in the following, the value of the Young modulus of fibers will be set to unity $E=1$. The constitutive behavior Eq. (3) of the bundle is perfectly linear up to the deformation $\varepsilon_{L}$ since no fibers break in this regime [see also Fig. 1(b)]. Due to the breaking of fibers above $\varepsilon_{L}$, the constitutive curve $\sigma(\varepsilon)$ becomes nonlinear and develops a maximum whose value $\sigma_{c}^{\mathrm{GLS}}$ and position $\varepsilon_{c}^{\mathrm{GLS}}$ define the failure stress and strain of the bundle, respectively. It follows from Eq. (3) that the critical strain is constant $\varepsilon_{c}^{\mathrm{GLS}}=1 / 2$ and does not depend on the cutoff strength $\varepsilon_{L}$, while $\sigma_{c}^{\mathrm{GLS}}$ increases due to the missing weak fibers [12]

$$
\sigma_{c}^{\mathrm{GLS}}=\frac{1}{4\left(1-\varepsilon_{L}\right)} .
$$

Increasing the external load quasistatically, the breaking fibers trigger avalanches of failure events which either stop after a finite fraction of fibers failed, or become unstable and destroy the entire system. As a consequence, the cutoff strength $\varepsilon_{L}$ can take meaningful values in the interval $0 \leqslant \varepsilon_{L} \leqslant \varepsilon_{c}^{\mathrm{GLS}}$, since for $\varepsilon_{L} \geqslant \varepsilon_{c}^{\mathrm{GLS}}$ the breaking of the first weakest fiber results in an immediate catastrophic failure of the bundle.

We explore the effect of the finite cutoff strength $\varepsilon_{L}$ on the failure process of FBMs with short ranged load sharing by means of computer simulations, redistributing the load of broken fibers according to the load transfer function Eq. (2). Stress controlled simulations were carried out on a square lattice of size $L=257$ with periodic boundary conditions varying the cutoff strength $\varepsilon_{L}$ of the disorder distribution Eq. (1) in the interval $[0,0.5]$ at several different values of the effective range of interaction $\gamma$ between 0 and 11. To characterize the failure process of the bundle at the macro and micro level, we determined the critical stress $\sigma_{c}$ and strain $\varepsilon_{c}$, the distribution $D$ of avalanche sizes $\Delta$, the average avalanche size $\langle\Delta\rangle$, and the average value of the largest avalanche $\left\langle\Delta_{\text {max }}\right\rangle$. For clarity, we first characterize the behavior of the system in the specific case of zero cutoff $\varepsilon_{L}=0$ by studying the critical stress $\sigma_{c}$ and strain $\varepsilon_{c}$ of the bundle as a function of $\gamma$, see Fig. 1(a). Based on the numerical results, three regimes of the failure of FBM can be distinguished in Fig. 1(a) depending on the range of load sharing: for $\gamma \leqslant 2$ the range of interaction is infinite in the two-dimensional embedding space, hence both $\sigma_{c}$ and $\varepsilon_{c}$ take their GLS values $\sigma_{c}=0.25$ and $\varepsilon_{c}=0.5$ independent of $\gamma$ [see Eq. (4) at $\left.\varepsilon_{L}=0\right]$. Increasing the value of $\gamma \geqslant 2$ the effective range of interaction gradually decreases, which lowers the macroscopic strength $\varepsilon_{c}$ and $\sigma_{c}$ of the bundle. In the limiting case of $\gamma \rightarrow \infty$ the model recovers the very localized load sharing, where $\varepsilon_{c}$ and $\sigma_{c}$ take again constant values. According to the numerical results, the perfectly localized limit is practically reached for $\gamma \geqslant 6$, so that in the interval $2 \leqslant \gamma \leqslant 6$ a transition occurs between the completely global and completely local behavior [11]. Figure 1(b) demonstrates that for $\gamma \leqslant 2$ (i.e., GLS) the macroscopic failure of the bundle is preceded by a strong nonlinearity of the constitutive curve $\sigma(\varepsilon)$. At any wider range of load sharing, $\gamma>2$, the $\sigma(\varepsilon)$ curves follow the GLS solution Eq. (3), but with lower strength values, which implies a more brittle macroscopic response for short ranged interactions.

Varying the cutoff strength $\varepsilon_{L}$ at different values of $\gamma$, it can be seen in Figs. 2(a) and 2(b) that in the long range regime $\gamma \leqslant 2$ both $\varepsilon_{c}$ and $\sigma_{c}$ agree well with the analytic predictions Eq. (4), i.e., $\varepsilon_{c}=1 / 2$ is constant while $\sigma_{c}$ increases with increasing cutoff $\varepsilon_{L}$. When the load sharing become short ranged $\gamma>2$, the increasing macroscopic brittleness has the consequence that the curves of $\varepsilon_{c}\left(\varepsilon_{L}\right)$ and $\sigma_{c}\left(\varepsilon_{L}\right)$ shift downwards as $\gamma$ increases and tend to a limit curve 


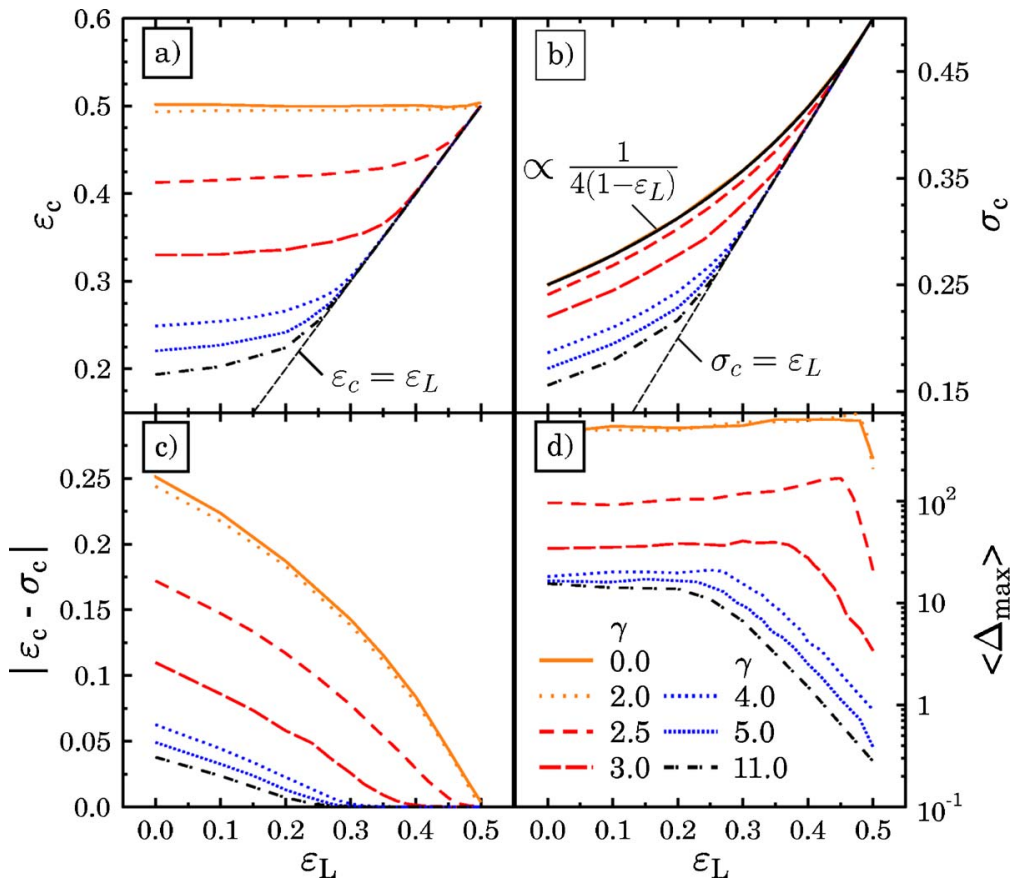

FIG. 2. (Color online) Characteristic quantities of the failure process of FBM varying the effective range of load sharing $\gamma$ and the cutoff value of failure strength $\varepsilon_{L}$ : (a) critical deformation $\varepsilon_{c}$, (b) critical stress $\sigma_{c}$, (c) $\left|\varepsilon_{c}-\sigma_{c}\right|$, (d) the average size of the largest avalanche $\left\langle\Delta_{\text {max }}\right\rangle$ as a function of $\varepsilon_{L}$ for various values of $\gamma$. when the interaction becomes completely localized for $\gamma \geqslant 6$. It is interesting to note that for short range interaction of fibers $\gamma>2$, not only the failure stress $\sigma_{c}$ but also the failure strain $\varepsilon_{c}$ is an increasing function of $\varepsilon_{L}$. It is important to emphasize that at each $\gamma$ there exists a critical value of the cutoff strength $\varepsilon_{L}^{c}<\varepsilon_{c}^{\mathrm{GLS}}$ where the failure stress $\sigma_{c}$ and strain $\varepsilon_{c}$ of the system becomes equal to the cutoff strength, i.e., at $\varepsilon_{L}^{c}$ holds $\varepsilon_{c}\left(\varepsilon_{L}^{c}\right)=\sigma_{c}\left(\varepsilon_{L}^{c}\right)=\varepsilon_{L}^{c}$. At this point the macroscopic response of the bundle becomes perfectly brittle, i.e., under gradual loading of the system the macroscopic constitutive behavior is linear up to $\sigma_{c}$, where the breaking of the weakest fiber gives rise to the collapse of the entire system [Fig. 1(b)]. This transition is better illustrated by Fig. 2(c) where the difference $\delta=\left|\varepsilon_{c}\left(\varepsilon_{L}\right)-\sigma_{c}\left(\varepsilon_{L}\right)\right|$ is plotted versus $\varepsilon_{L}$. It can be observed that $\delta$ monotonically decreases and becomes practically zero at $\varepsilon_{L}^{c}$ of the given $\gamma$. Since the absence of weak fibers gives rise to a higher macroscopic strength, the value of $\varepsilon_{L}^{c}$ is larger than the strength of the bundle $\varepsilon_{c}$ and $\sigma_{c}$ at zero cutoff [see Fig. 1(a)].

On the microlevel, the failure process is characterized by the bursts of fiber breakings, which also show an interesting behavior when the range of interaction $\gamma$ and the lower cutoff $\varepsilon_{L}$ are varied. In the GLS regime $\gamma<2$, our computer simulations perfectly recover the analytical and numerical results of Refs. $[9,10]$ [see Fig. 3(a)]: for $\varepsilon_{L}=0$ the size distribution of bursts $D(\Delta)$ follows a power law

$$
D(\Delta) \sim \Delta^{-\alpha},
$$

with an exponent $\alpha=5 / 2$. Increasing the value of the cutoff $\varepsilon_{L}$, for small avalanches, a crossover occurs to a power law of a lower exponent $\alpha=3 / 2$, while for large avalanches the original power law with $\alpha=5 / 2$ is retained. The crossover to a lower value of the exponent indicates that, due to the missing weak fibers, the fraction of small avalanches decreases compared to the larger ones. This argument is further sup- ported by Fig. 2(d) and Fig. 4 which demonstrate that for $\gamma \leqslant 2$ both the average size of the largest avalanche $\left\langle\Delta_{\max }\right\rangle$ and the average avalanche size $\langle\Delta\rangle$ are monotonically increasing functions of the cutoff $\varepsilon_{L}$. However, when the load sharing gets short ranged $\gamma>2$, both $\left\langle\Delta_{\max }\right\rangle$ and $\langle\Delta\rangle$ have a maximum at the critical cutoff strength. The qualitative behavior of the crossover avalanche size $\Delta_{c}$ is equal to that of $\langle\Delta\rangle$ : for $\gamma>2, \Delta_{c}$ has a maximum at $\epsilon_{L}^{c}$, and the height of the maximum decreases with increasing $\gamma$. Note that the nonvanishing avalanche size above $\varepsilon_{L}^{c}$ arises due to the strength fluctuations of the finite bundle so that above $\varepsilon_{L}^{c}$ the bundle

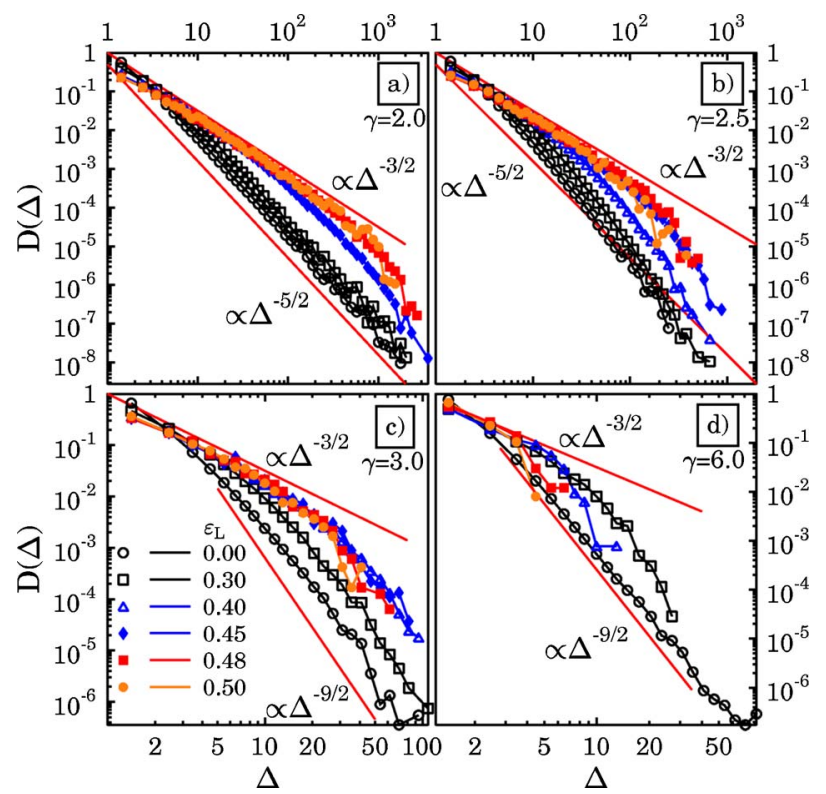

FIG. 3. (Color online) Distribution of burst sizes $D(\Delta)$ varying $\varepsilon_{L}$ at different values of $\gamma$ : (a) 2.0, (b) 2.5, (c) 3.0, (d) 6.0. Crossover behavior of $D(\Delta)$ can be observed as $\varepsilon_{L}$ approaches the critical cutoff value $\varepsilon_{L}^{c}(\gamma)$. 


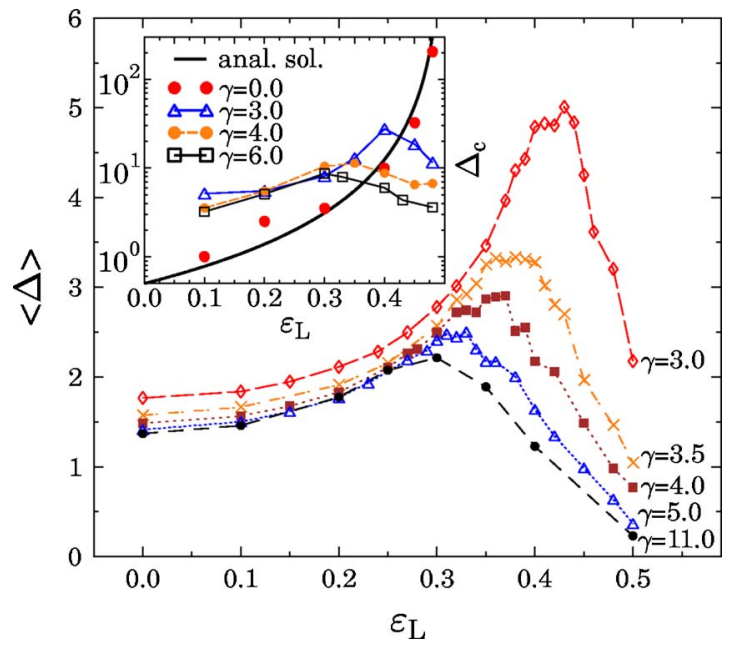

FIG. 4. (Color online) The average burst size $\langle\Delta\rangle$ as a function of $\varepsilon_{L}$ for different values of $\gamma$. In the short range regime $\gamma>2$, the average avalanche size $\langle\Delta\rangle$ has a clear maximum that coincides with the critical cutoff strength $\varepsilon_{L}^{c}$. Inset, crossover avalanche size $\Delta_{c}$ and GLS analytical solution [13].

may survive a small number of avalanches instead of collapsing after the breaking of the weakest fiber. It is interesting to note that contrary to GLS, in the transition regime $2<\gamma<6$, the avalanche size distribution does not show a power law behavior for small cutoffs $\varepsilon_{L} \approx 0$, however, when $\varepsilon_{L}$ approaches the critical value $\varepsilon_{L}^{c}(\gamma)$, the distribution of burst sizes $D(\Delta)$ tends again to a power law of an exponent $\alpha=3 / 2$ [Figs. 3(a) and 3(b)]. For very localized interactions $\gamma>6$ an apparent power law of $D(\Delta)$ is restored for $\varepsilon_{L} \approx 0$ with a relatively high exponent $\alpha \approx 9 / 2$, in agreement with Ref. [8] [Fig. 3(d)]. The main outcome of computer simulations is that the crossover behavior of $D(\Delta)$ to the universal power law $D(\Delta) \sim \Delta^{-3 / 2}$ prevails at any value of the range of interaction $\gamma$ for the limiting case of $\varepsilon_{L} \rightarrow \varepsilon_{L}^{c}(\gamma)$, independently of the original form of $D(\Delta)$ at zero cutoff $\varepsilon_{L}=0$ (see Fig. 3). In spite of the relatively large system size $L$, for short range interaction of fibers and $\varepsilon_{L} \rightarrow \varepsilon_{L}^{c}(\gamma)$ the statistics of avalanche sizes is rather poor for large avalanches, which hinders us from making a definite conclusion on the shape of $D(\Delta)$ in this $\Delta$ regime.

An interesting experimental realization of the crossover for crackling noise was very recently found in the magnitude distribution of earthquakes in Japan [14]. Analyzing the local magnitude distribution of earthquakes preceding main shocks, a significant decrease of the Gutenberg-Richter exponent was obtained when the lower bound of the time window of the analysis is shifted towards the catastrophic event [14]. Fracture of ferromagnetic materials is accompanied by changes of the magnetic flux, which can be recorded as magnetic noise and provides information on the dynamics of crack propagation [15]. The amplitude, area, and energy of magnetic emission signals have recently been found to have power law distributions with exponents depending on the type of fracture, i.e., ductile failure where stable crack propagation occurs in a large number of elementary steps is characterized by significantly higher exponents than brittle failure, where the crack propagates in an unstable catastrophic manner breaking the specimen in a few large jumps [15]. Our numerical results suggest that the reduction of nonlinearity of the macroscopic response of materials preceding global failure when going from ductile and quasibrittle to brittle fracture is responsible for the lowering of the crackling noise exponents on the microlevel.

In summary, we carried out computer simulations of the failure process of a bundle of fibers with a finite cutoff of the fibers' strength, continuously varying the range of interaction between the limiting cases of global and local load sharing. We showed that increasing the cutoff strength $\varepsilon_{L}$ the macroscopic response of the fiber bundle becomes perfectly brittle when $\varepsilon_{L}$ approaches a critical value $\varepsilon_{L}^{c}(\gamma)$, depending on the range of interaction $\gamma$. Our numerical results demonstrate the robustness of the crossover of the avalanche size distribution $D(\Delta)$ to a universal power law of exponent $3 / 2$, irrespective of the range of interaction between the material elements.

This work was supported by the Collaborative Research Center SFB381. F. Kun acknowledges financial support of the Research Contracts Nos. NKFP-3A/043/04, OTKA M041537, and T049209.
[1] A. Garcimartín, A. Guarino, L. Bellon, and S. Ciliberto, Phys. Rev. Lett. 79, 3202 (1997).

[2] A. Guarino, A. Garcimartin, and S. Ciliberto, Eur. Phys. J. B 6, 13 (1998).

[3] A. Petri, G. Paparo, A. Vespignani, A. Alippi, and M. Costantini, Phys. Rev. Lett. 73, 3423 (1994).

[4] R. C. Hidalgo, F. Kun, and H. J. Herrmann, Phys. Rev. E 64, 066122 (2001).

[5] S. Zapperi, P. Ray, H. E. Stanley, and A. Vespignani, Phys. Rev. Lett. 78, 1408 (1997).

[6] J. V. Andersen, D. Sornette, and K. T. Leung, Phys. Rev. Lett. 78, 2140 (1997).

[7] M. Kloster, A. Hansen, and P. C. Hemmer, Phys. Rev. E 56, 2615 (1997).
[8] A. Hansen and P. C. Hemmer, Phys. Lett. A 184, 394 (1994).

[9] S. Pradhan, A. Hansen, and P. C. Hemmer, Phys. Rev. Lett. 95, 125501 (2005).

[10] S. Pradhan and A. Hansen, Phys. Rev. E 72, 026111 (2005).

[11] R. C. Hidalgo, Y. Moreno, F. Kun, and H. J. Herrmann, Phys. Rev. E 65, 046148 (2002).

[12] S. Pradhan, P. Bhattacharyya, and B. K. Chakrabarti, Phys. Rev. E 66, 016116 (2002).

[13] S. Pradhan, A. Hansen, and P. C. Hemmer, Phys. Rev. E 74, 016122 (2006).

[14] H. Kawamura, e-print cond-mat/0603335.

[15] F. Kun, G. B. Lenkey, N. Takács, and D. L. Beke, Phys. Rev. Lett. 93, 227204 (2004). 